\title{
Upgrading of
}

\section{Traditional Electric Meter}

\section{Into Wireless Electric Meter}

\author{
Using ZigBee Technology \\ Berhanu Regassa, Verónica Medina, Isabel M. Gómez, Octavio Rivera, \\ and José A. Gómez \\ Escuela Técnica Superior de Ingeniería Informática \\ Dpto. de Tecnología Electrónica \\ Avda. Reina Mercedes s/n, 41014 Sevilla, Spain \\ \{ berregbal,vmedina,igomez, jgomezdte\}@us.es, octavio@dte.us.es
}

\begin{abstract}
Since the electric generating, distributing and marketing has been possible; the Electric Meter was introduced and developed along the years, but never like this time. The digital technology, the wireless communication, and the computer systems have dramatically changed the Electric meter generation from Traditional Meter to Automatic Meter and now to Smart Meter. Their differences lie in how to collect and process information, and the amount of information they can provide. Even so the traditional meter reading has been long used, and it is still one of the most common ways for meter reading. This method, however, has their disadvantages which are the result of human basis reading method that uses. If the reading problem is solved by adding a simple circuit, which makes it automatic it will be still useful method of reading. In this paper it is described a prototype for Automatic Meter Reading (AMR) system that uses a Traditional meter, ZigBee modules, and Mesh network. As result the system gives, full capability of automatic meter even some features of smart meter too. This experiment has an important result for developing country like Ethiopia, which almost $99 \%$ its meters are the Traditional meters.
\end{abstract}

Keywords: ZigBee, WSN, AMR, eZeeNet.

\section{Introduction}

\subsection{Meter Backgrounds}

Electrical meters have been used from the late 19th century [1]. First meters operated on liquid movements, analogous to sand watch. Then Traditional meters have arrived, surviving without major changes for more than 100 years. Traditional meters are changing in electronic, highly computerized environment of both electricity production and distribution, and customer service and payment processing.

Actually Traditional meters, either they are being replaced or they are being upgraded. A few major disadvantages are clear: 
- A great number of inspectors have to be employed for reading the meters.

- Sending out bills is an expensive procedure.

- Processing payments is even more expensive, requiring a large number of employees.

- Single tariff constrains effective usage of electricity. Power distribution companies can't discourage peak time usage by punitive tariff and promote night-time consumption by industrial customers with lower tariff.

AMR not only overcomes these obstacles but also offer a number of additional advantages:

- AMR meters could be connected in network, just like computers.

- Real-time reading gives up-to-the-minute clear picture of consumption with any details required, even up to single building level. Power Distribution Company now knows where to upgrade cables and transformers. It doesn't have to rely any more on subjective requests of its customers or use often misleading monthly averages.

- Prepaid cards let avoid any bad debts.

- Customer is automatically disconnected when his prepaid balance reaches zero.

- Not only multi-tariffs are allowed but they also could be changed remotely at any time.

\subsection{Communication technology}

The AMR [13] [14] [15] [16] [17] system requires means of communication for transmitting and receiving their data. The information collected from each meter, must arrive reliably and securely to the utility provider, for billing and analyzing. In another way any command from provider office that addresses each node and each meter must arrive reliably and securely to its destination. In the network of nodemeters each node and meter are identified by their unique id (Node-Id and Meter-Id).

The selection of which communication technology [2] to use depends on a complex set of engineering analysis that requires significant expertise and experience in communications, together with an in-depth knowledge of the application and its requirements. No single technology is the best price and performance solution for every application.

From the broadest list of communication technology such as PLC, GSM, Wi-Fi, ZigBee [11][12] and others, the ones that more are used in AMR are the GSM and ZigBee.

GSM (Global System for Mobile Communication) is a development from cellular telephony. GSM adopts digital modulation and the key technology is time division multiple access (TDMA).

ZigBee [3] is a two-way wireless communication technology featuring short distance, low complexity, low power consumption, low data speed, and low cost. It is used mainly in data exchange between low power electronic devices within a short range. There can be as many as 65,000 wireless communication modules in a ZigBee network, which is very similar to telecommunication networks like CDMA or GSM. 
Each ZigBee module is like a telecommunication station, and the modules can communicate with each other within the whole network. The communication distances between the nodes can range from the standard 75 meters to hundreds of meters and even several kilometres. The ZigBee network can also be connected to other networks.

\subsection{Advantages and Disadvantages}

The main advantage of GSM, with its good anti-piracy ability, large network capacity, high reliability and high sensitivity, for that it can provide many data transmission services. Its main disadvantage is high cost.

ZigBee is the most popular industry wireless mesh networking standard for connecting sensors, actuators, and instrumentation and control systems.

The main advantages of ZigBee are:

- Power saving, as a result of the short working period, low power consumption of communication, and standby mode.

- Reliability: Collision avoidance is adopted, with a special time slot allocated for those communications that need fixed bandwidth so that competition and conflict are avoided when transmitting data. The MAC layer adopts completely confirmed data transmission, that is, every data packet sent must wait for the confirmation from the receiver.

- Low cost of the modules and the ZigBee protocol is patent fee free.

- Short time delay, typically $30 \mathrm{~ms}$ for device searching, $15 \mathrm{~ms}$ for standby to activation, and $15 \mathrm{~ms}$ for channel access of active devices.

- Large network capacity: One ZigBee network contains one master device and maximum 65,000 slave devices. There can be as many as 100 ZigBee networks within one area.

- Safety: ZigBee provides a data integrity check and authentication function. AES128 is adopted and at the same time each application can flexibly determine its safety property.

\section{System overview}

Normally the AMR infrastructure includes hardware, software, communications, customer associated systems and meter data management software, supplier and network distribution business systems.

This prototype of upgrading focuses on designing a suitable interface circuit that makes the traditional meter functions exactly the same as, even better than Automatic Meter. For that purpose it has designed with economical circuit that uses component such as Current transformer sensor [4] which permits to read the information from the cable that enters to the meter, and power devices which controls the power.

Each meter contains an Interface Circuit (IC) and a ZigBee module according to the connection type. Practically there are two types of connection regarding the ZigBee module. A group-meters-connection when various meters are connected to a 
module and a single-meter-connection when a meter is connected to a module. Mainly the group-meters-connection reduces various ZigBee modules; as consequence it reduces the total cost of modules and reduces also network traffic or load.

The network connection, which is interconnected via mesh, involves End device, routers, coordinators, concentrators, range extender and ZigBee-GSM coordinator according to the ZigBee standard [3]. End-devices collect data from Interface circuit, transfer data and receive commands to control the meter. For any reason that the supplier wants to break the power from his client, the module can switch-on and switch-off the line that enters to the meter. Router passes the data from and to near-by modules. The coordinator serves as gateway, transferring data to other coordinator or to central office. In case the coordinator is located too far for normal link, the special module ZigBee-GSM is used. The module contains both ZigBee and GSM circuits. The packet data coming from ZigBee mesh network passes to GSM to be sent to central office through GSM network. In the office side a similar module receives the data and transfers to the server.

\section{System Design}

The prototype design consists of three elements: Electromechanical or Traditional meter, Interface circuit, and ZigBee modules (Fig. 1 shows the interconnection). The essential work is: the design of economic interface circuit and the programming of ZigBee modules. The modules will be programmed to act according to the standards of the ZigBee wireless network the same as End-Device, Router, and Coordinator, also as Concentrator, and Range extender.

\subsection{Electromechanical or Traditional meter}

The electromechanical induction meter operates by counting the revolutions of an aluminum disc which is made to rotate at a speed proportional to the power. The number of revolutions is thus proportional to the energy usage. The aluminum disc is supported by a spindle which has a worm gear which drives the register. The register is a series of dials which record the amount of energy used. Most domestic electricity meters must be read manually, whether by a representative of the power company or by the customer. This prototype aims to free the tedious job of reading by making it automatic. First: Convert the power usage into digital data; which is accomplished by interface circuit. Second: transmit the read data via ZigBee networks that ZigBee modules will be in charge. Third: received data will be processed according their use at office.

\subsection{Interface Circuit Design}

Most electricity meters measure current (amperes) and voltage (volts) and calculate the product of these values to determine electrical power (watts). Power integrated 
over time then provides the energy that is used (typically expressed as watt hours or joules).

There are various methods for sensing the amount of current flowing in a wire. They are: Shunt resistor, Current transformer, Hall Effect, Magneto resistor sensor, and Fiber optics sensor. [5] In order to measure the current and volt in this Interface Circuit is used the Current-sense transformers. They have been long the standard for precise current measurements in instrumentation applications. They are accurate, good isolation, easy to implement, reliable under harsh environmental and thermal conditions.

The Interface Circuit design has two parts: the first one is for measuring the current and the voltage, and the second is the controlling part. In measuring part it is used the current transformer AC1005 for current sensing. The component supports current load up to $60 \mathrm{~A}$, big enough to measure a broad range of current. The controlling part contain: the Triac with Optocoupler for switching on/off the power.

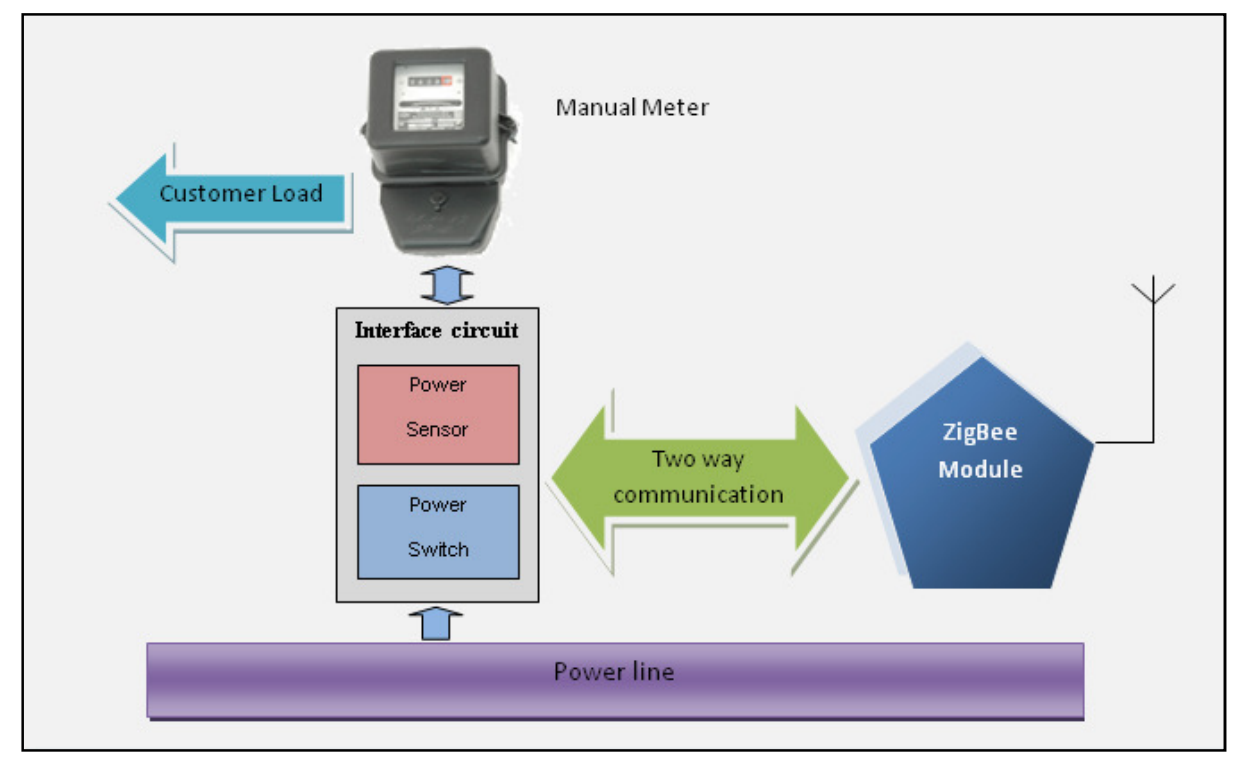

Fig. 1. Block diagram of Electric meter connection (Meter, Interface circuit, and ZigBee module, which is an End-Device). 


\subsection{Modules or network members}

A crucial question is what kind of devices or network members are taking part in an AMR; On the ZigBee network level it is clear: Each network consists of one coordinator and many end devices and routers. As for the application level, we've identified the following device categories that form AMR network. See Fig. 2.

- Traditional or Manual meter: the device used to measure the electric consumption posted by the utility company in each house.

- Interface circuit: It is an intermediate device between the meter, the communication device, and the power line; the same is responsible for reading the power consumption that pass through the cable that goes to the manual meter and also controls the power flow that enters to the meter. That mean when it is necessary it may break the electric flow that goes to the meter.

- ZigBee-End-Device: collects and keeps updating data coming from interface circuit; and on request, it passes data to immediate router or concentrator. There are two types of connections between Meters and End-Devices: One, a group of meters can be connected to a single End-device (Fig. 3), for example one building (if, only the meters are located nearby area). Two, when a house is found alone could also be connected with a single Meter through an End-Device.

- ZigBee-router: pass data coming from or to other End-Devices to immediate router or coordinator.

- ZigBee-Coordinator and GSM: This top hierarchy device is a top collector of all data coming from subordinated routers and establish the far connection with the service provider office via GSM. The module contains both ZigBee and GSM circuits. The coming data will be packed and passed to GSM circuit for sending, then the GSM establish the connection and transmits the packet of data in form of SMS to an office for billing or analyzing. The collector of data through GSM/SMS will be in the central office; in charge of receiving all the data coming from different ZigBee-coordinators.

- $\quad$ Range Extender: A stand alone device with ZigBee routing functionality.

- Concentrator: Is able to collect, store and forward metering data from other meters. Concentrators are mainly thought to manage metering data from meters that are not always online.

\subsection{Networking}

The system uses two types of networks. ZigBee Mesh networks to collect the meters reading and GSM network to take the collected data of meters via ZigBee to an Electric Office. 99\% the network is covered by ZigBee, while GSM network become as a closer-gap; the distance that can not covered by ZigBee will be filled by GSM. It is a bridge or a gateway of collected data to an office. 
In the network formation, the concentrator collects meter data from meters via enddevices attached to each meters, also passes commands to end-devices, for reading and identification purpose. The range extender closes the gap when there is a big street, park or anything very wide, between concentrator, router o coordinator devices. For range extender it is used an amplified module of ZigBee, the module has a transmission range of $4 \mathrm{~km}$ in open air.

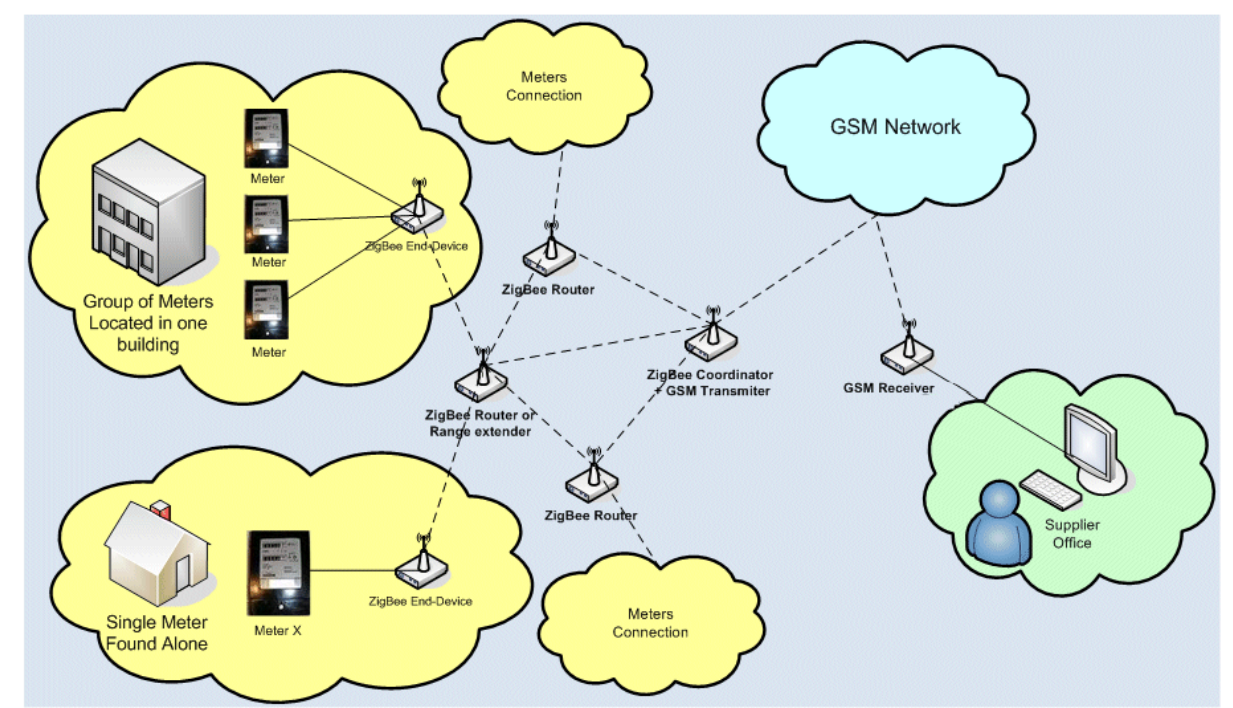

Fig. 2. AMR network overview.

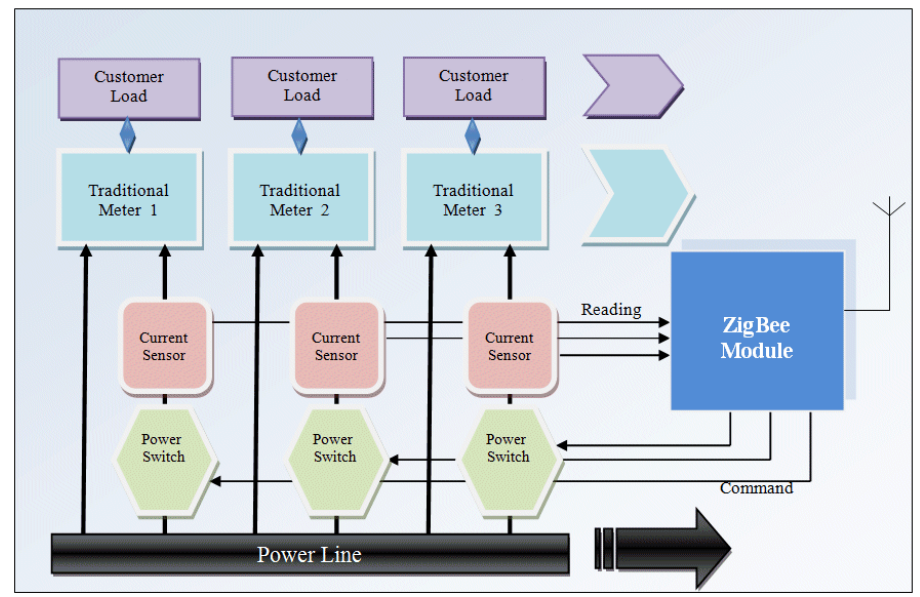

Fig. 3 A group of meter connection controlled by a ZigBee Module. 


\subsection{Hardware components list}

The lists of components that are necessary to build the system are as follow:

- ZigBee development boards, to act as different required devices for the system, i.e., End-Device, Concentrator, Range extender, Router, and Coordinator) from Meshnetics[18].

- AC1005 current transformer sensor.

- GSM module (for too far connection)

- A power semiconductor Triacs.

- Optocoupler.

\subsection{ZigBee Development Board}

The development boards [6] are based on ZigBit, ZigBit Amp and ZigBit 900 RF module with a matched antenna. Antenna options include PCB antenna and external SMA connector, as well as on-module integrated chip antenna. Powered by two AA batteries, the standard board, the amplified board and the latest development boards come all one preset with 2 frequently used sensors - temperature and illumination, as well as a number of other peripheral devices. The boards also support extension connector allowing easy access to the module's interfaces.

ZigBit boards contain Atmel's ATmega1281V Microcontroller [7] and AT86RF230 RF transceiver the module features $128 \mathrm{~K}$ bytes flash memory and $8 \mathrm{~K}$ bytes RAM.

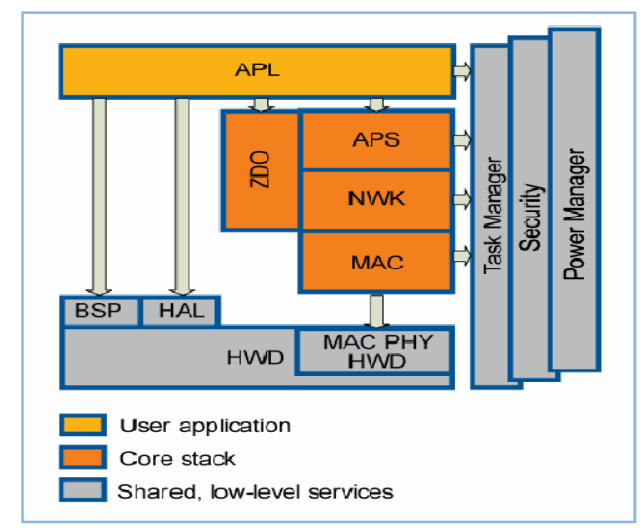

Fig. 4. eZeeNet Software Stack Architecture.

\subsection{Software design}

The ZigBit module ships with robust 802.15 .4 /ZigBee stack that supports a selfhealing, self-organizing mesh network, while optimizing network traffic and minimizing power consumption. The modules have three stack configurations: 
eZeeNet, SerialNet and OpenMAC. Each of them has their own purpose, in this case it is used the eZeeNet (Fig. 4) [8].

The eZeeNet Stack conforms to ZigBee specification and it can be easily reconfigured to drive End-Device, Router or Coordinator. Easy-to-use interfaces simplify basic network operations, namely data exchange, network formation/node join, PAN ID management, channel selection, TX power control, and more.

The software design apart from creating the network and assure the proper function, allows to send commands and to make the meter readings. The eZeeNet stack is responsible for formation and management of networks, but the commands and the meter readings need to be specified and also formatted the meters' data.

The main commands are such as:

- Read Meter Data: order the meter reading.

- Switch on/off specific meter: order either on or off, it is a remote on/off command.

- Node scanning: the command to check if all nodes are working correctly.

The fig. 5 shows user interface prototype that is used to communicate with ZigBee network of meters in two way communication.

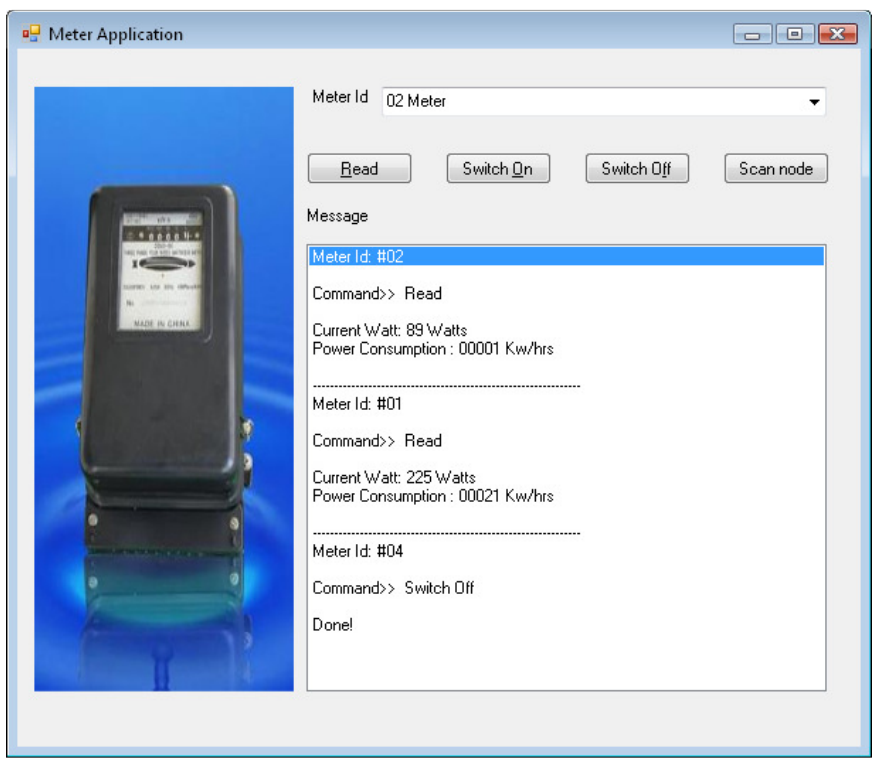

Fig. 5 Meter application user interface prototype.

The meter data format can be Node Id, Meter Id, and Reading (KWh, ratio). Even though it is known that the stack uses its own node identification logic, which is the Mac address; here the AMR system sets other identification: Meter Id and Node Id, for better flexibility and easy management of the network resources. 


\subsection{Testing result of measurement}

The boards that form the AMR network are tested; whereas the testing point has been the IC. The following Table 1 shows a simple experiment of bulbs with different watts, showing sensed amperes, and converted into digital to feed the MCU for an applied voltage of $230 \mathrm{~V}$.

\begin{tabular}{|r|l|l|r|}
\hline Bulb in Watts & \multicolumn{1}{|c|}{ ADC } & Volt & \multicolumn{1}{c|}{ Ampere } \\
\hline 160 & 73.00 & 0.0892 & 0.892 \\
\hline 120 & 54.00 & 0.0660 & 0.660 \\
\hline 100 & 43.00 & 0.0525 & 0.525 \\
\hline 60 & 26.00 & 0.0318 & 0.318 \\
\hline 40 & 16.00 & 0.0196 & 0.196 \\
\hline
\end{tabular}

Table 1 Experiment results Interface circuit.

\section{Conclusions}

This working prototype of ZigBee based AMR system is to demonstrate the upgrading made to traditional meter is very effective. It has low infrastructure cost, simple and easy installation that resolves the reading problems of manual meters. Cost wise they are reasonable comparing to others type wireless technology. Billing and customer data management system is required that the system becomes complete, but it is out of the scope of this project.

Even though, it is known that in developed country the electric meters are substituting the traditional meters with digital meters commercially available [9][10], which are based on different technologies (ZigBee, PLC, GSM, and others); in developing country like Ethiopia, country origin to one of the authors, the proposed solution in this prototype is much cheaper. 


\section{References}

1. http://watthourmeters.com/history.html

2. Networking fundamentals: wide, local and personal area communications /Kaveh Pahlavan, Prashant Krishnamurthy ISBN 9780470992906

3. ZigBee Alliance, www.zigbee.org

4. Nuvotem Talema http://www.talema-nuvotem.com/en/products/as.shtml

5. Instrumentación Electrónica, Miguel A. Pérez García, Juan C. Álvarez Antón, Juan C. Campo Rodríguez, Fco. Javier Ferrero Martin, Gustavo J. Grillo Ortega. ISBN 8497321669

6. ZigBit Development Kit Users Guide, MeshNetics Doc. S-ZDK-451 01 v.1.10.

7. Atmel Corporation- (http://www.atmel.com/ products/zigbee/zigbit_modules.asp?family_id=676).

8. eZeeNet ${ }^{\mathrm{TM}}$ IEEE802.15.4/ZigBee Software. Product Datasheet. MeshNetics Doc. M-251 02.

9. Holley Metering Limited - (http://www.holleymeter.com/en/index.php).

10. Wasion Group Limited - (http://wasionmeters.en.alibaba.com/).

11.Leveraging the Fuññ Potentila of Automated Meter Reading (AMR) Technologies, GCI Group Inc, 2004.

12. Practical Results of AMR Codecs Exploitation in GSM network, Ivica Radovic,1, Borivoj Modlic2 49th International Symposium ELMAR-2007, 12-14 September 2007, Zadar, Croatia Network planning department, T-Mobile, Vukovarska 23, Zagreb, Croatia pp 107-11010.

13. Networked Wireless Meter Reading System Based on ZigBee Technology Liting Cao, Wei Jiang, Zhaoli Zhang, 2008 IEEE. 978-1-4244-1734-6/08/c 2008 IEEE pp 3455-3460.

14. Automatic Power Meter Reading System Using GSM Network H.G.Rodney Tan,IEEE, C.H. Lee and V.H. Mok, IEEE. 978-981-05-9423-7_c 2007 RPS pp 465-469.

15. "ZigBee Technology and Its Application on Wireless Meter-reading System," Bo Chen; Mingguang Wu; Shuai Yao; Ni Binbin; , Industrial Informatics, 2006 IEEE International Conference on , vol., no., pp.1257-1260, 16-18 Aug. 2006 doi: 10.1109/INDIN.2006.275820

16. "Automatic Meter Reading System Based on Wireless Mesh Networks and SOPC Technology," Liting Cao; Wei Jiang; Zhaoli Zhang; , Intelligent Networks and Intelligent Systems, 2009. ICINIS '09. Second International Conference on, vol., no., pp.142-145, 1-3 Nov. 2009 doi: 10.1109/ICINIS.2009.45

17. "Realization of Automatic Meter Reading System Based on ZigBee with Improved Routing Protocol,"

Guilin Zheng; Yichuan Gao; Lijuan Wang; , Power and Energy Engineering Conference (APPEEC), 2010 Asia-Pacific , vol., no., pp.1-6, 28-31 March 2010 doi: 10.1109/APPEEC.2010.5448276

18. http://www.meshnetics.com/ 